TRANSACTIONS OF THE

AMERICAN MATHEMATICAL SOCIETY

Volume 349, Number 7, July 1997, Pages 2901-2916

S 0002-9947(97)01721-2

\title{
ON THE FABER COEFFICIENTS OF FUNCTIONS UNIVALENT IN AN ELLIPSE
}

\author{
E. HALILOGLU
}

Abstract. Let $E$ be the elliptical domain

$$
E=\left\{x+i y: \frac{x^{2}}{(5 / 4)^{2}}+\frac{y^{2}}{(3 / 4)^{2}}<1\right\} .
$$

Let $S(E)$ denote the class of functions $F(z)$ analytic and univalent in $E$ and satisfying the conditions $F(0)=0$ and $F^{\prime}(0)=1$. In this paper, we obtain global sharp bounds for the Faber coefficients of the functions $F(z)$ in certain related classes and subclasses of $S(E)$.

\section{INTRODUCTION}

After the proof of the Bieberbach Conjecture, the interest in coefficient problems in certain classes of functions analytic in the unit disc has decreased. In this paper, we are interested in the problem of finding bounds for coefficients of some classes of functions $F(z)$ in domains $\Omega$ other than the unit disc; specifically, we will consider an elliptical domain. For this purpose we must have a series expansion of $F(z)$ which is, in certain ways, a generalization of the Taylor expansion. The Faber expansion of $F(z)$ in $\Omega$ will serve this purpose.

The results obtained in this study are related to the results for the unit disc. Therefore it will be convenient to recall some well-known coefficient estimates for certain classes of functions analytic in the unit disc.

Let $S$ denote the class of functions $f(z)$ which are analytic and univalent in the unit disc $\mathbf{D}=\{z:|z|<1\}$, and normalized so that

$$
f(z)=z+\sum_{n=2}^{\infty} a_{n} z^{n} \quad(|z|<1) .
$$

Let $C$ denote the subclass of $S$ consisting of functions $f(z)$ for which $f(\mathbf{D})$ is a convex set. Functions in $C$ are called convex functions.

A function $f(z)$ analytic in $\mathbf{D}$ is said to be typically real if and only if $f(z)$ is real for $-1<z<1$. We denote the class of such functions with the conditions $f(0)=0$ and $f^{\prime}(0)=1$ by $T$.

Let $P$ denote the class of functions $p(z)$ analytic in $\mathbf{D}$ with $p(0)=1$ and $\operatorname{Re}\{p(z)\}>0$ for $z \in \mathbf{D}$.

Let $S^{(2)}$ denote the class of odd functions in $S$.

Received by the editors October 17, 1994 and, in revised form, January 22, 1996.

1991 Mathematics Subject Classification. Primary 30C45; Secondary 33C45.

Key words and phrases. Faber polynomials, Faber coefficients, Chebyshev polynomials, Jacobi elliptic sine function.

(C) 1997 American Mathematical Society 
In 1917, Loewner [9] showed that if $f(z) \in C$ is given by (1), then

$$
\left|a_{n}\right| \leq 1 \quad(n=2,3, \cdots)
$$

and the inequality is sharp. He showed that equality is attained only for functions of the form

$$
c_{\theta}(z)=\frac{z}{1-e^{i \theta} z}, \quad \theta \in[0,2 \pi) .
$$

In 1907, Carathéodory [5] showed that if

$$
p(z)=1+\sum_{n=1}^{\infty} b_{n} z^{n} \in P,
$$

then

$$
\left|b_{n}\right| \leq 2 \quad(n=1,2, \cdots) .
$$

This result is sharp. The functions

$$
p_{\theta}(z)=\frac{1+e^{i \theta} z}{1-e^{i \theta} z}, \quad \theta \in[0,2 \pi),
$$

are examples of functions for which equality is obtained in (4).

In 1931, Rogosinski [11] proved that if $f(z) \in T$ is given by (1), then

$$
\left|a_{n}\right| \leq n \quad(n=2,3, \cdots) .
$$

Equality occurs for all even $n$ if $f(z)$ is the Koebe function

$$
k(z)=\frac{z}{(1-z)^{2}}
$$

or $-k(-z)$. Equality occurs for all odd $n$ if $f(z)$ is a convex combination of $k(z)$ and $-k(-z)$.

In 1916, Bieberbach [1] conjectured that the inequality given by (6) holds for the entire class $S$. The Bieberbach conjecture was proved by de Branges in 1984 [2]. de Branges also proved that equality holds in (6) only if $f(z)$ is a rotation of the Koebe function, i.e.

$$
f(z)=e^{-i \theta} k\left(e^{i \theta} z\right), \quad \theta \in[0,2 \pi) .
$$

We next introduce the Faber polynomials associated with a simply connected domain $\Omega$. Let $\Omega$ be a bounded, simply connected domain in $\mathbb{C}$ for which $0 \in \Omega$. Let $g(z)$ be the unique, one-to-one, analytic mapping of $\Delta=\{z:|z|>1\}$ onto $\mathbb{C} \backslash \bar{\Omega}$ with

$$
g(z)=c z+\sum_{n=0}^{\infty} \frac{c_{n}}{z^{n}} \quad(c>0,|z|>1) .
$$

Assume that $\Omega$ has capacity 1 , so that $c=1$ in (8). The Faber polynomials $\left\{\Phi_{n}(z)\right\}_{n=0}^{\infty}$ associated with $\Omega$ (or $g(z)$ ) are defined by the generating function relation $[6, \mathrm{p} .218]$

$$
\frac{w g^{\prime}(w)}{g(w)-z}=\sum_{n=0}^{\infty} \Phi_{n}(z) w^{-n}
$$


It is known [12, p.42] that if $F(z)$ is analytic in $\operatorname{Int} \Omega$ and $\partial \Omega$ is analytic then $F(z)$ has the representation

$$
F(z)=\sum_{n=0}^{\infty} A_{n} \Phi_{n}(z), \quad z \in \operatorname{Int} \Omega
$$

where

$$
A_{n}=\frac{1}{2 \pi i} \int_{|z|=\rho} F(g(z)) z^{-n-1} d z
$$

with $\rho<1$ and close to 1 . Also the series in (10), which is called the Faber series, converges uniformly on compact subsets of Int $\Omega$. Thus the Faber polynomials provide an important tool in studying functions analytic in a simply connected domain $\Omega$.

We define $S(\Omega)$ to be the class of functions $F(z)$ which are analytic and univalent in $\Omega$ and normalized by the conditions $F(0)=0$ and $F^{\prime}(0)=1$. If $\varphi(z)$ is the unique, one-to-one, analytic mapping of $\Omega$ onto $\mathbf{D}$ with $\varphi(0)=0$ and $\varphi^{\prime}(0)>0$, then $F(z) \in S(\Omega)$ if and only if

$$
F(z)=\frac{f(\varphi(z))}{\varphi^{\prime}(0)}
$$

for some $f(z) \in S$. The Faber series (10) of $F(z)$ will be written as

$$
F(z)=\sum_{n=0}^{\infty} A_{n}(f) \Phi_{n}(z)
$$

where $f(z) \in S$ is the function characterized by (11).

It is not easy to deal with both exterior and interior mappings at the same time, so we restrict our interest to an elliptical domain, for which both of these functions are manageable. We will concentrate on the domain $\Omega=E$, where

$$
E=\left\{x+i y: \frac{x^{2}}{(5 / 4)^{2}}+\frac{y^{2}}{(3 / 4)^{2}}<1\right\} .
$$

The function $g(z)=z+\frac{1}{4 z}$ is a one-to-one, analytic mapping of $\Delta$ onto $\mathbb{C} \backslash \bar{E}$. Substituting $z+\frac{1}{4 z}$ for $g(z)$ in (9) and carrying out necessary algebra yield that the Faber polynomials $\left\{\Phi_{n}(z)\right\}_{n=0}^{\infty}$ associated with $E$ are the monic Chebyshev polynomials, given by

$$
\begin{gathered}
\Phi_{n}(z)=2^{-n}\left\{\left[z+{\sqrt{z^{2}-1}}^{n}+\left[z-{\sqrt{z^{2}-1}}^{n}\right\} \quad(n=1,2, \cdots),\right.\right. \\
\Phi_{0}(z)=1 .
\end{gathered}
$$

Now let $\operatorname{sn}(z ; q)$ be the Jacobi elliptic sine function with nome $q$ and modulus $0<k_{0} \leq 1$, and let

$$
K=\int_{0}^{1} \frac{1}{\sqrt{1-t^{2}} \sqrt{1-k_{0}^{2} t^{2}}} d t
$$

(see [8, Chap. 2]). Then the function

$$
\varphi(z)=\sqrt{k_{0}} \operatorname{sn}\left(\frac{2 K}{\pi} \sin ^{-1} z ; \frac{1}{16}\right)
$$


is the one-to-one, analytic mapping of $E$ onto $\mathbf{D}$ which satisfies the conditions $\varphi(0)=0$ and $\varphi^{\prime}(0)=\frac{2 K \sqrt{k_{0}}}{\pi}>0[10$, p.296].

We now define classes of functions on $E$ analogous to the special classes associated with $\mathbf{D}$ as follows:

$$
C(E)=\{F(z) \in S(E): F(E) \text { is convex }\}
$$

and

$$
S^{(2)}(E)=\{F(z) \in S(E): F(z) \text { is odd }\} .
$$

Also, let $T(E)$ denote the class of functions $F(z)$ analytic in $E$ with the properties $F(0)=0, F^{\prime}(0)=1$ and $F(z)$ has real values for $-\frac{5}{4}<z<\frac{5}{4}$ and nonreal values elsewhere. Finally, let $P(E)$ denote the class of functions $P(z)$ analytic in $E$ with $P(0)=\frac{1}{\varphi^{\prime}(0)}=\frac{\pi}{2 K \sqrt{k_{0}}}$ and $\operatorname{Re}\{P(z)\}>0$. (The condition $P(0)=\frac{1}{\varphi^{\prime}(0)}$ is imposed for convenience.)

Functions $F(z)$ in the classes $C(E)$ and $P(E)$ may be expressed in the form (11) for functions $f(z)$ in the classes $C$ and $P$, respectively. Since $\varphi(z)$ is odd and $-1<\varphi(z)<1$ for $-\frac{5}{4}<z<\frac{5}{4}$, the functions $F(z)$ in the classes $S^{(2)}(E)$ and $T(E)$ may be expressed in the form (11) for some functions $f(z)$ in the classes $S^{(2)}$ and $T$, respectively.

Let the Faber expansions of functions $F(z)$ in the classes indicated above be given by (12). In this paper, we obtain sharp bounds for the Faber coefficients $\left\{A_{n}(f)\right\}_{n=0}^{\infty}$ of functions $F(z)$ in the classes $C(E), T(E)$ and $P(E)$. We also show that equalities hold only if $f(z)=c_{0}(z)=\frac{z}{1-z}$ or $f(z)=c_{\pi}(z)=\frac{z}{1+z}, f(z)=k(z)$ or $f(z)=$ $-k(-z)$ where $k(z)$ is the Koebe function given by $(7)$, and $f(z)=p_{0}(z)=\frac{1+z}{1-z}$ or $f(z)=p_{\pi}(z)=\frac{1-z}{1+z}$, respectively. Hence, in all three cases there are two extremal functions, which is equivalent to the number of rotations of the ellipse. In addition, we make a conjecture for the Faber coefficients of functions in the class $S(E)$.

\section{Main Results}

Lemma. If $F(z)$ is analytic in $E$ and has the Faber series given by (10), then the Faber coefficients $\left\{A_{n}\right\}_{n=0}^{\infty}$ are given by

$$
A_{n}=\frac{2^{n}}{\pi} \int_{0}^{\pi} F(\cos \theta) \cos n \theta d \theta \quad(n=0,1,2, \cdots)
$$

Proof. We have from (10)

$$
F(\cos \theta)=\sum_{n=0}^{\infty} A_{n} \Phi_{n}(\cos \theta) .
$$

Substituting $\Phi_{n}(\cos \theta)=2^{1-n} \cos n \theta$ into (13) gives

$$
F(\cos \theta)=\sum_{n=0}^{\infty} A_{n} 2^{1-n} \cos n \theta
$$

Multiplying (14) by $\cos m \theta$ and then integrating from 0 to $\pi$ completes the proof of the lemma. 
Corollary 1. If the functions in the classes $S(E), C(E), S^{(2)}(E)$, and $P(E)$ have the Faber expansions given by (12), then the Faber coefficients $\left\{A_{n}(f)\right\}_{n=0}^{\infty}$ are given by

$$
A_{n}(f)=\frac{2^{n}}{2 K \sqrt{k_{0}}} \int_{0}^{\pi} f(\varphi(\cos \theta)) \cos n \theta d \theta, \quad(n=0,1,2, \cdots) .
$$

Proof. Corollary 1 follows from the lemma and the relation (11), since $\varphi^{\prime}(0)=$ $\frac{2 K \sqrt{k_{0}}}{\pi}$.

In the next corollary we show that for functions in $S^{(2)}(E)$, the even Faber coefficients are 0 .

Corollary 2. If $F(z) \in S^{(2)}(E)$, then $A_{2 n}(f)=0 \quad(n=0,1,2, \cdots)$.

Proof. From (15) we have

$$
A_{2 n}(f)=\frac{2^{2 n}}{2 K \sqrt{k_{0}}} \int_{0}^{\pi / 2}[f(\varphi(\cos \theta))+f(\varphi(-\cos \theta))] \cos 2 n \theta d \theta \quad(n=0,1,2, \cdots) .
$$

Thus $A_{2 n}(f)=0 \quad(n=0,1,2, \cdots)$, because both $f(z)$ and $\varphi(z)$ are odd functions.

Let $\mathcal{F}$ denote one of the sets $C, T$, and $P$. Then $\mathcal{F}$ is a compact set. Hence the closed convex hull of $\mathcal{F}, \overline{\mathrm{co}}(\mathcal{F})$, is also compact, and, since $A_{n}(f)$ is a continuous linear functional,

$$
M=\max _{f \in \overline{c o}(\mathcal{F})}\left|A_{n}(f)\right|
$$

exists. In addition, we have

$$
\max _{f \in \mathcal{F}}\left|A_{n}(f)\right|=\max _{\operatorname{ext}(\overline{\mathrm{co}}(\mathcal{F}))}\left|A_{n}(f)\right| .
$$

The extreme points of $\overline{\mathrm{co}}(C)$ and $\overline{\mathrm{co}}(T)$ are determined in [4] as follows:

$$
\operatorname{ext}(\overline{\mathrm{co}}(C))=\left\{f: f(z)=c_{\theta}(z), \quad 0 \leq \theta<2 \pi\right\}
$$

and

$$
\operatorname{ext}(\overline{\mathrm{CO}}(T))=\left\{f: f(z)=t_{\theta}(z), \quad 0 \leq \theta \leq \pi\right\}
$$

where $c_{\theta}(z)$ is given by $(2)$ and

$$
t_{\theta}(z)=\frac{z}{1-2 z \cos \theta+z^{2}}
$$

The extreme points of $\overline{\mathrm{co}}(P)[3]$ are given by

$$
\operatorname{ext}(\overline{\mathrm{co}}(P))=\left\{f: f(z)=p_{\theta}(z), \quad 0 \leq \theta<2 \pi\right\},
$$

where $p_{\theta}(z)$ is given by (5). Using (16) with (17), (18), and (20), we see that the problem of maximizing $\left|A_{n}(f)\right|$ over the classes $C, T$, and $P$ reduces to the problem of maximizing the values of $\left|A_{n}\left(c_{\theta}\right)\right|(\theta \in[0,2 \pi)),\left|A_{n}\left(t_{\theta}\right)\right|(\theta \in[0, \pi])$, and $\left|A_{n}\left(p_{\theta}\right)\right|$ $(\theta \in[0,2 \pi))$ over $\theta$, respectively.

In the following theorems we evaluate the values of $A_{n}\left(c_{\theta}\right), A_{n}\left(t_{\theta}\right)$, and $A_{n}\left(p_{\theta}\right)$, where $A_{n}(f)$ is given by (15). We need to use different countours for different quadrants of $\theta$. So each theorem includes one quadrant of $\theta$. 
Theorem 1. If $c_{\theta}(z)$ is given by (2), then

$$
\begin{aligned}
A_{n}\left(c_{\theta}\right)= & \frac{\pi^{2} e^{-i \theta}\left(e^{i n \alpha(\theta)}-2^{-2 n} e^{-i n \alpha(\theta)}\right)}{4 K^{2} \sqrt{k_{0}}\left(1-2^{-4 n}\right)\left(1+k_{0}^{2}-2 k \cos 2 \theta\right)^{1 / 2}}, \\
& 0 \leq \theta \leq \frac{\pi}{2} \quad(n=0,1,2, \cdots),
\end{aligned}
$$

where $0 \leq \alpha(\theta) \leq \frac{\pi}{2}$ is given by

$$
\varphi\left[\cos \left(\alpha(\theta)+\frac{\pi \tau}{4}\right)\right]=e^{-i \theta}, \quad 0 \leq \theta \leq \frac{\pi}{2} \quad \text { with } \tau=\frac{4 i \ln 2}{\pi} .
$$

Proof. The function $\cos z$ maps the rectangle $R$ with vertices at the points $-\frac{\pi \tau}{4}$, $\pi-\frac{\pi \tau}{4}, \pi+\frac{\pi \tau}{4}$, and $\frac{\pi \tau}{4}$ onto $E$. Therefore the function $\varphi(\cos z)$ maps $R$ onto $\Delta(0,1)$ with

$$
\varphi\left[\cos \left(\alpha(t)+\frac{\pi \tau}{4}\right)\right]=e^{-i t}, \quad 0 \leq t \leq \frac{\pi}{2},
$$

where $\alpha(t)$ increases from 0 to $\frac{\pi}{2}$ as $t$ increases from 0 to $\frac{\pi}{2}$.

Integrate the function $h(z)=c_{\theta}(\varphi(\cos z)) e^{i n z}$ over the parallelogram $A B C D$ with vertices at the points $-\pi, \pi, \pi \tau$, and $\pi \tau-2 \pi$, respectively. From (21) we see that $\alpha(\theta)+\frac{\pi \tau}{4}$ is a pole of $h(z)$ inside $A B C D$.

Let $i K^{\prime}=K \tau$, and refer to $\operatorname{sn}\left(z ; \frac{1}{16}\right)$ as $\operatorname{sn} z$ for convenience. Then

$$
\varphi(\cos (\pi \tau-z))=\sqrt{k_{0}} \operatorname{sn}\left(\frac{2 K}{\pi}\left(\frac{\pi}{2}-\pi \tau+z\right)\right)=\sqrt{k_{0}} \operatorname{sn}\left(\frac{2 K}{\pi}\left(\frac{\pi}{2}+z\right)\right),
$$

since $\operatorname{sn} z$ is doubly periodic with periods $2 i K^{\prime}$ and $4 K$. Thus

$$
\varphi(\cos z)=\varphi(\cos (-z))=\varphi(\cos (\pi \tau-z)) .
$$

It follows from $(22)$ that $-\alpha(\theta)+\frac{3 \pi \tau}{4}$ is the other pole of $h(z)$ inside $A B C D$. So by the residue theorem

$$
\oint_{A B C D} h(z) d z=2 \pi i\left(\operatorname{Res}_{\alpha(\theta)+\frac{\pi \tau}{4}}+\operatorname{Res}_{-\alpha(\theta)+\frac{3 \pi \tau}{4}}\right)
$$

where $\operatorname{Res}_{z_{0}}$ denotes the residue of the function $h(z)$ at the point $z=z_{0}$.

The contribution of the integrals on $B C$ and $D A$ cancel each other because $h(z)$ is a periodic function with period $2 \pi$. Now

$$
\int_{A B} h(z) d z=\int_{-\pi}^{\pi} h(x) d x=2 \int_{0}^{\pi} c_{\theta}(\varphi(\cos x)) \cos n x d x
$$

and

$$
\int_{C D} h(z) d z=\int_{2 \pi}^{0} h(x+\pi \tau-2 \pi) d x=-\int_{0}^{2 \pi} h(x+\pi \tau) d x .
$$

From (22) we obtain

$$
\begin{aligned}
\int_{C D} h(z) d z & =-\int_{0}^{2 \pi} e^{i n(x+\pi \tau)} c_{\theta}(\varphi(\cos x)) d x \\
& =2 \cdot 2^{-4 n} \int_{0}^{\pi} c_{\theta}(\varphi(\cos x)) \cos n x d x .
\end{aligned}
$$


Then adding (24) and (25) results in

$$
\oint_{A B C D} h(z) d z=2\left(1-2^{-4 n}\right) \int_{0}^{\pi} c_{\theta}(\varphi(\cos x)) \cos n x d x .
$$

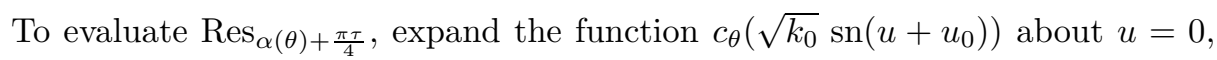
where

$$
u_{0}=\frac{2 K}{\pi}\left(\frac{\pi}{2}-\alpha(\theta)-\frac{\pi \tau}{4}\right) .
$$

The addition formula for sn $u[8, \mathrm{p} .33]$ yields

$$
\sqrt{k_{0}} \operatorname{sn}\left(u+u_{0}\right)=\frac{\sqrt{k_{0}} \operatorname{sn} u \operatorname{cn} u_{0} \operatorname{dn} u_{0}+\sqrt{k_{0}} \operatorname{sn} u_{0} \operatorname{cn} u \operatorname{dn} u}{1-k_{0}^{2} \operatorname{sn}^{2} u_{0} \operatorname{sn}^{2} u}
$$

where $\operatorname{cn} z$ and $\operatorname{dn} z$ refer to $\operatorname{cn}\left(z ; \frac{1}{16}\right)$ and $\operatorname{dn}\left(z ; \frac{1}{16}\right)$, respectively. It follows from (21) that

$$
\sqrt{k_{0}} \text { sn } u_{0}=e^{-i \theta}, \quad 0 \leq \theta \leq \frac{\pi}{2} .
$$

To evaluate $\operatorname{cn} u_{0}$ and $\operatorname{dn} u_{0}$, employ the identities

$$
\mathrm{sn}^{2} z+\mathrm{cn}^{2} z=1
$$

and

$$
k_{0}^{2} \operatorname{sn}^{2} z+\operatorname{dn}^{2} z=1
$$

[8, p.25]. To determine whether to use the + or $-\operatorname{sign}$ for $\operatorname{cn} u_{0}$ and $\operatorname{dn} u_{0}$, check the signs of $\operatorname{Re}\left\{\operatorname{cn}\left(x-\frac{i K^{\prime}}{2}\right)\right\}$ and $\operatorname{Re}\left\{\operatorname{dn}\left(x-\frac{i K^{\prime}}{2}\right)\right\}$, respectively. Deduce from the addition formulas for $\operatorname{cn} u$ and $\operatorname{dn} u[8, \mathrm{p} .34]$ that

$$
\operatorname{cn}\left(x-\frac{i K^{\prime}}{2}\right)=\sqrt{\frac{1+k_{0}}{k_{0}}} \frac{\operatorname{cn} x+i \operatorname{sn} x \operatorname{dn} x}{1+k_{0} \operatorname{sn}^{2} x}
$$

and

$$
\operatorname{dn}\left(x-\frac{i K^{\prime}}{2}\right)=\frac{\sqrt{1+k_{0}}\left(\operatorname{dn} x+i k_{0} \operatorname{sn} x \operatorname{cn} x\right)}{1+k_{0} \operatorname{sn}^{2} x} .
$$

Thus $\operatorname{Re}\left\{\operatorname{cn}\left(x-\frac{i K^{\prime}}{2}\right)\right\} \geq 0$ and $\operatorname{Re}\left\{\operatorname{dn}\left(x-\frac{i K^{\prime}}{2}\right)\right\} \geq 0$ for $x \in[0, K]$, since cn $x$ decreases from 1 to 0 and $\operatorname{dn} x$ decreases from 1 to $\sqrt{1-k_{0}^{2}}$ for $x \in[0, K]$. Hence using (29) and (30) we obtain

$$
\operatorname{cn} u_{0}=\sqrt{1-\frac{e^{-2 i \theta}}{k_{0}}}
$$

and

$$
\operatorname{dn} u_{0}=\sqrt{1-k_{0} e^{-2 i \theta}} .
$$

Choosing the principal branch as $-\pi<\arg z \leq \pi$, we obtain

$$
0 \leq \arg \left(\operatorname{cn} u_{0}\right) \leq \frac{\pi}{2}
$$

and

Therefore

$$
0 \leq \arg \left(\operatorname{dn} u_{0}\right) \leq \frac{\pi}{4}
$$

$$
0 \leq \arg \left(\operatorname{cn} u_{0} \operatorname{dn} u_{0}\right) \leq \frac{3 \pi}{4}
$$


which implies

$$
\sqrt{k_{0}} \operatorname{cn} u_{0} \operatorname{dn} u_{0}=i e^{-i \theta}\left(1+k_{0}^{2}-2 k_{0} \cos 2 \theta\right)^{1 / 2}
$$

Using

$$
\begin{gathered}
\operatorname{sn} u=u-\frac{1}{3 !}\left(1+k_{0}^{2}\right) u^{3}+\cdots, \\
\operatorname{cn} u=1-\frac{1}{2 !} u^{2}+\cdots, \\
\operatorname{dn} u=1-\frac{1}{2 !} k_{0}^{2} u^{2}+\cdots
\end{gathered}
$$

[8, p.37], and (31) in (28) and doing necessary calculations, we get

$$
\sqrt{k_{0}} \operatorname{sn}\left(u+u_{0}\right)=e^{-i \theta}+i e^{-i \theta}\left(1+k_{0}^{2}-2 k_{0} \cos 2 \theta\right)^{1 / 2} u+\cdots .
$$

Thus

or

$$
c_{\theta}\left(\sqrt{k_{0}} \operatorname{sn}\left(u+u_{0}\right)\right)=\frac{i e^{-i \theta}}{\left(1+k_{0}^{2}-2 k_{0} \cos 2 \theta\right)^{1 / 2} u}+\cdots
$$

$c_{\theta}\left(\sqrt{k_{0}} \operatorname{sn}\left(\frac{2 K}{\pi}\left(\frac{\pi}{2}-z\right)\right)\right)=-\frac{\pi i e^{-i \theta}}{2 K\left(1+k_{0}^{2}-2 k_{0} \cos 2 \theta\right)^{1 / 2}\left(z-\alpha(\theta)-\frac{\pi \tau}{4}\right)}+\cdots$.

Hence we obtain

$$
\operatorname{Res}_{\alpha(\theta)+\frac{\pi \tau}{4}}=-\frac{\pi i e^{-i \theta} 2^{-n} e^{i n \alpha(\theta)}}{2 K\left(1+k_{0}^{2}-2 k_{0} \cos 2 \theta\right)^{1 / 2}}
$$
as

In a similar way, the residue of $h(z)$ at the point $-\alpha(\theta)+\frac{3 \pi \tau}{4}$ may be obtained

$$
\operatorname{Res}_{-\alpha(\theta)+\frac{3 \pi \tau}{4}}=\frac{\pi i e^{-i \theta} 2^{-3 n} e^{-i n \alpha(\theta)}}{2 K\left(1+k_{0}^{2}-2 k_{0} \cos 2 \theta\right)^{1 / 2}} .
$$

Substituting (36) and (37) into (23) yields

$$
\oint_{A B C D} h(z) d z=\frac{\pi^{2} e^{-i \theta} 2^{-n}}{K\left(1+k_{0}^{2}-2 k_{0} \cos 2 \theta\right)^{1 / 2}}\left(e^{i n \alpha(\theta)}-2^{-2 n} e^{-i n \alpha(\theta)}\right) .
$$

Comparing (26) and (38) gives the desired result.

For $\theta$ in other quadrants, proofs are similar to the proof of Theorem 1. Therefore we will state the theorems and then in the proofs indicate only the integration countours and poles of $h(z)$ inside the countours.

Theorem 2. If $c_{\theta}(z)$ is given by (2), then

$$
\begin{gathered}
A_{n}\left(c_{\theta}\right)=\frac{(-1)^{n} \pi^{2} e^{-i \theta}\left(e^{-i n \alpha(\pi-\theta)}-2^{-2 n} e^{i n \alpha(\pi-\theta)}\right)}{4 K^{2} \sqrt{k_{0}}\left(1-2^{-4 n}\right)\left(1+k_{0}^{2}-2 k_{0} \cos 2 \theta\right)^{1 / 2}}, \\
\frac{\pi}{2} \leq \theta \leq \pi \quad(n=0,1,2, \cdots)
\end{gathered}
$$

where $\alpha(\theta)$ is as in Theorem 1. 
Proof. Integrate the function $h(z)=c_{\theta}(\varphi(\cos z)) e^{i n z}$ over the parallelogram $A B C D$ with vertices at the points $0,2 \pi, 3 \pi+\pi \tau, \pi+\pi \tau$. Inside $A B C D$ there are two poles of $h(z)$, at the points $\pi-\alpha(\pi-\theta)+\frac{\pi \tau}{4}$ and $\pi+\alpha(\pi-\theta)+\frac{3 \pi \tau}{4}$.

Theorem 3. If $c_{\theta}(z)$ is given by (2), then

$$
\begin{gathered}
A_{n}\left(c_{\theta}\right)=\frac{(-1)^{n} \pi^{2} e^{-i \theta}\left(e^{i n \alpha(\theta-\pi)}-2^{-2 n} e^{-i n \alpha(\theta-\pi)}\right)}{4 K^{2} \sqrt{k_{0}}\left(1-2^{-4 n}\right)\left(1+k_{0}^{2}-2 k_{0} \cos 2 \theta\right)^{1 / 2}}, \\
\pi \leq \theta \leq \frac{3 \pi}{2} \quad(n=0,1,2, \cdots)
\end{gathered}
$$

where $\alpha(\theta)$ is as in Theorem 1.

Proof. Integrate the function $h(z)=c_{\theta}(\varphi(\cos z)) e^{i n z}$ over the parallelogram $A B C D$ with vertices at the points $0,2 \pi, 3 \pi-\pi \tau$, and $\pi-\pi \tau$. Inside $A B C D$ there are two poles of $h(z)$, at the points $\pi-\alpha(\theta-\pi)-\frac{\pi \tau}{4}$ and $\pi+\alpha(\theta-\pi)-\frac{3 \pi \tau}{4}$.

Theorem 4. If $c_{\theta}(z)$ is given by (2), then

$$
\begin{gathered}
A_{n}\left(c_{\theta}\right)=\frac{\pi^{2} e^{-i \theta}\left(e^{-i n \alpha(2 \pi-\theta)}-2^{-2 n} e^{i n \alpha(2 \pi-\theta)}\right)}{4 K^{2} \sqrt{k_{0}}\left(1-2^{-4 n}\right)\left(1+k_{0}^{2}-2 k_{0} \cos 2 \theta\right)^{1 / 2}}, \\
\frac{3 \pi}{2} \leq \theta \leq 2 \pi \quad(n=0,1,2, \cdots),
\end{gathered}
$$

where $\alpha(\theta)$ is as in Theorem 1 .

Proof. Integrate the function $h(z)=c_{\theta}(\varphi(\cos z)) e^{i n z}$ over the parallelogram $A B C D$ with vertices at the points $-\pi, \pi,-\pi \tau$, and $-2 \pi-\pi \tau$. Two poles of $h(z)$ occur at the points $\alpha(2 \pi-\theta)-\frac{\pi \tau}{4}$ and $-\alpha(2 \pi-\theta)-\frac{3 \pi \tau}{4}$.

Theorem 5. If $p_{\theta}(z)$ is given by (5), then

$$
A_{n}\left(p_{\theta}\right)=2 A_{n}\left(c_{\theta}\right), \quad 0 \leq \theta<2 \pi \quad(n=0,1,2, \cdots) .
$$

The proof is similar to the proofs of Theorems 1-4.

Since the function

$$
t_{\theta}(z)=\frac{z}{1-2 z \cos \theta+z^{2}}, \quad 0 \leq \theta \leq \pi,
$$

has double poles at the points $\theta=0$ and $\theta=\pi$, we will treat these cases separately. (Note that for $\theta=0, t_{\theta}(z)$ becomes the Koebe function.)

Theorem 6. If $k(z)$ is given by (7), then

$$
A_{n}(k)=\frac{\pi^{3} n}{8 K^{3} \sqrt{k_{0}}(1-k)^{2}\left(1-2^{-2 n}\right)} \quad(n=1,2, \cdots) .
$$

Proof. Integrate the function $h(z)=k(\varphi(\cos z)) e^{i n z}$ over the countour used in the proof of Theorem 1. Inside the parallelogram $A B C D$, there are two double poles of $h(z)$ at the points $\frac{\pi \tau}{4}$ and $\frac{3 \pi \tau}{4}$. So by the residue theorem

$$
\oint_{A B C D} h(z) d z=2 \pi i\left(\operatorname{Res} \frac{\pi \tau}{4}+\operatorname{Res} \frac{3 \pi \tau}{4}\right) .
$$


As in Theorem 1,

$$
\oint_{A B C D} h(z) d z=2\left(1-2^{-4 n}\right) \int_{0}^{\pi} k(\varphi(\cos x)) \cos n x d x .
$$

To find $\operatorname{Res} \frac{\pi \tau}{4}$, expand $k\left(\sqrt{k_{0}} \operatorname{sn}\left(u+K-\frac{i K^{\prime}}{2}\right)\right)$ about $u=0$. Doing the necessary calculations, we obtain

$$
k\left(\sqrt{k_{0}} \operatorname{sn}\left(\frac{2 K}{\pi}\left(\frac{\pi}{2}-z\right)\right)\right)=-\frac{\pi^{2}}{4 K^{2}\left(1-k_{0}\right)^{2}\left(z-\frac{\pi \tau}{4}\right)^{2}}+\frac{0}{\left(z-\frac{\pi \tau}{4}\right)}+\cdots .
$$

Writing

$$
e^{i n z}=2^{-n} e^{i n\left(z-\frac{\pi \tau}{4}\right)}=2^{-n}\left[1+i n\left(z-\frac{\pi \tau}{4}\right)+\cdots\right]
$$

and multiplying (41) by (42) yields

$$
\operatorname{Res} \frac{\pi \tau}{4}=\frac{-i \pi^{2} n 2^{-n}}{4 K^{2}\left(1-k_{0}\right)^{2}} .
$$

In a similar way, the residue of $h(z)$ at $z=\frac{3 \pi \tau}{4}$ is obtained as

$$
\operatorname{Res}_{\frac{3 \pi \tau}{4}}=-2^{-3 n} \text { in } \frac{-i \pi^{2} n 2^{-3 n}}{4 K^{2}\left(1-k_{0}\right)^{2}} .
$$

Substituting (43) and (44) into (39) yields

$$
\oint_{A B C D} h(z) d z=\frac{\pi^{3} n 2^{-n}\left(1+2^{-2 n}\right)}{2 K^{2}\left(1-k_{0}\right)^{2}} .
$$

Equating (40) and (45) and solving for $A_{n}(k)$ gives the desired result.

Theorem 7. If $t_{\theta}(z)$ is given by (19), then

$$
A_{n}\left(t_{\pi}\right)=\frac{(-1)^{n-1} \pi^{3} n}{8 K^{3} \sqrt{k_{0}}\left(1-k_{0}\right)^{2}\left(1-2^{-2 n}\right)} \quad(n=1,2, \cdots) .
$$

The proof is similar to the proof of Theorem 6 .

Theorem 8. If $t_{\theta}(z)$ is given by (19), then

$$
\begin{gathered}
A_{n}\left(t_{\theta}\right)=\frac{\pi^{2} \sin n \alpha(\theta)}{4 K^{2} \sqrt{k_{0}}\left(1-2^{-2 n}\right) \sin \theta\left(1+k_{0}^{2}-2 k_{0} \cos 2 \theta\right)^{1 / 2}}, \\
0<\theta \leq \frac{\pi}{2} \quad(n=1,2, \cdots),
\end{gathered}
$$

where $\alpha(\theta)$ is as in Theorem 1 .

Proof. Integrate the function $h(z)=t_{\theta}(\varphi(\cos z)) e^{i n z}$ over the rectangle $P Q R S$ with vertices at the points $-\pi, \pi,-\pi+\pi \tau$, and $-\pi+\pi \tau$. The poles of $h(z)$ occur at the points $z$ for which

$$
\varphi(\cos z)=e^{-i \theta}, \quad 0<\theta \leq \frac{\pi}{2}
$$

and

$$
\varphi(\cos z)=e^{i \theta}, \quad 0<\theta \leq \frac{\pi}{2} .
$$


We found in Theorem 1 that solutions of (46) inside $P Q R S$ are $\alpha(\theta)+\frac{\pi \tau}{4}$ and $-\alpha(\theta)+\frac{3 \pi \tau}{4}$. Similarly, solutions of (47) inside PQRS are obtained as $-\alpha(\theta)+\frac{\pi \tau}{4}$ and $\alpha(\theta)+\frac{3 \pi \tau}{4}$. So by the residue theorem

$$
\oint_{P Q R S} h(z) d z=2 \pi i\left(\operatorname{Res}_{\alpha(\theta)+\frac{\pi \tau}{4}}+\operatorname{Res}_{-\alpha(\theta)+\frac{3 \pi \tau}{4}}+\operatorname{Res}_{-\alpha(\theta)+\frac{\pi \tau}{4}}+\operatorname{Res}_{\alpha(\theta)+\frac{3 \pi \tau}{4}}\right) .
$$

By periodicity of $h(z)$ the integrals over $Q R$ and $S P$ cancel each other. We have

$$
\int_{P Q} h(z) d z=2 \int_{0}^{\pi} t_{\theta}(\varphi(\cos x)) \cos n x d x
$$

and

$$
\int_{R S} h(z) d z=\int_{\pi}^{-\pi} t_{\theta}(\varphi(\cos (x+\pi \tau))) e^{i n(x+\pi \tau)} d x .
$$

Using (22) in (50) gives

$$
\int_{R S} h(z) d z=-2 \cdot 2^{-4 n} \int_{0}^{\pi} t_{\theta}(\varphi(\cos x)) \cos n x d x .
$$

Adding (49) and (51) yields

$$
\oint_{P Q R S} h(z) d z=2\left(1-2^{-4 n}\right) \int_{0}^{\pi} t_{\theta}(\varphi(\cos x)) \cos n x d x .
$$

It follows from (35) that

$$
t_{\theta}\left(\sqrt{k_{0}} \operatorname{sn}\left(u+u_{0}\right)\right)=\frac{1}{2 \sin \theta\left(1+k_{0}^{2}-2 k_{0} \cos 2 \theta\right)^{1 / 2}}+\cdots,
$$

where $u_{0}$ is given by (27). Hence

$$
\operatorname{Res}_{\alpha(\theta)+\frac{\pi \tau}{4}}=-\frac{\pi 2^{-n} e^{i n \alpha(\theta)}}{4 K \sin \theta\left(1+k_{0}^{2}-2 k_{0} \cos 2 \theta\right)^{1 / 2}} .
$$

To find $\operatorname{Res}_{-\alpha(\theta)+\frac{3 \pi \tau}{4}}$, expand the function $t_{\theta}\left(\sqrt{k_{0}} \operatorname{sn}\left(v+v_{0}\right)\right)$ about $v=0$, where

$$
v_{0}=\frac{2 K}{\pi}\left(\frac{\pi}{2}+\alpha(\theta)-\frac{3 \pi \tau}{4}\right) .
$$

The addition formula for the Jacobi elliptic sine function gives

$$
\sqrt{k_{0}} \operatorname{sn}\left(v+v_{0}\right)=\frac{\sqrt{k_{0}}\left(\operatorname{sn} v \operatorname{cn} v_{0} \operatorname{dn} v_{0}+\operatorname{sn} v_{0} \operatorname{cn} v \operatorname{dn} v\right)}{1-k_{0}^{2} \operatorname{sn}^{2} v_{0} \operatorname{sn}^{2} v} .
$$

We have

$$
\sqrt{k_{0}} \operatorname{sn} v_{0}=e^{i \theta}, \quad 0<\theta \leq \frac{\pi}{2} .
$$

By using addition formulas for $\operatorname{cn} u$ and $\operatorname{dn} u$ we can easily show that

$$
\operatorname{Re}\left\{\operatorname{cn}\left(x-\frac{3 i K^{\prime}}{2}\right)\right\} \geq 0
$$

and

$$
\operatorname{Re}\left\{\operatorname{dn}\left(x-\frac{3 i K^{\prime}}{2}\right\} \leq 0\right.
$$


for $K<x \leq 2 K$. Hence it follows from (29) and (30) that

$$
\operatorname{cn} v_{0}=\sqrt{1-\frac{e^{-2 i \theta}}{k_{0}}}
$$

and

$$
\operatorname{dn} v_{0}=-\sqrt{1-k_{0} e^{-2 i \theta}}
$$

respectively. Therefore (31) results in

$$
\sqrt{k_{0}} \operatorname{cn} v_{0} \operatorname{dn} v_{0}=-i e^{-i \theta}\left(1+k_{0}^{2}-2 k_{0} \cos 2 \theta\right)^{1 / 2} .
$$

Using (32), (33), (34), and (55) in (54) and doing some manipulation, we get

$$
\sqrt{k_{0}} \operatorname{sn}\left(v+v_{0}\right)=e^{-i \theta}-i e^{-i \theta}\left(1+k_{0}^{2}-2 k_{0} \cos 2 \theta\right)^{1 / 2} v+\cdots .
$$

Thus

$$
t_{\theta}\left(\sqrt{k_{0}} \operatorname{sn}\left(v+v_{0}\right)\right)=-\frac{1}{2 \sin \theta\left(1+k_{0}^{2}-2 k_{0} \cos 2 \theta\right)^{1 / 2} v}+\cdots .
$$

As a result,

$$
\operatorname{Res}_{-\alpha(\theta)+\frac{3 \pi \tau}{4}}=\frac{\pi 2^{-3 n} e^{-i n \alpha(\theta)}}{4 K \sin \theta\left(1+k_{0}^{2}-2 k_{0} \cos 2 \theta\right)^{1 / 2}} .
$$

Choosing a principal branch, we obtain

$$
\sqrt{k_{0}-e^{2 i \theta}} \sqrt{1-k_{0} e^{2 i \theta}}=-i e^{i \theta}\left(1+k_{0}^{2}-2 k_{0} \cos 2 \theta\right)^{1 / 2} .
$$

Then using the above argument with (57) gives

$$
\operatorname{Res}_{-\alpha(\theta)+\frac{\pi \tau}{4}}=\frac{\pi 2^{-n} e^{-i n \alpha(\theta)}}{4 K \sin \theta\left(1+k_{0}^{2}-2 k_{0} \cos 2 \theta\right)^{1 / 2}}
$$

and

$$
\operatorname{Res}_{-\alpha(\theta)+\frac{3 \pi \tau}{4}}=-\frac{\pi 2^{-3 n} e^{i n \alpha(\theta)}}{4 K \sin \theta\left(1+k_{0}^{2}-2 k_{0} \cos 2 \theta\right)^{1 / 2}} .
$$

Substituting (53), (56), (58), and (59) into (48) yields

$$
\oint_{P Q R S} h(z) d z=\frac{\pi^{2} 2^{-n}\left(1+2^{-2 n}\right) \sin n \alpha(\theta)}{K \sin \theta\left(1+k_{0}^{2}-2 k_{0} \cos 2 \theta\right)^{1 / 2}} .
$$

Equating (52) and (60) gives the desired result.

Theorem 9. If $t_{\theta}(z)$ is given by (19), then

$$
\begin{gathered}
A_{n}\left(t_{\theta}\right)=\frac{(-1)^{n-1} \pi^{2} \sin n[\alpha(\pi-\theta)]}{4 K^{2} \sqrt{k_{0}}\left(1-2^{-2 n}\right) \sin \theta\left(1+k_{0}^{2}-2 k_{0} \cos 2 \theta\right)^{1 / 2}}, \\
\frac{\pi}{2} \leq \theta<\pi \quad(n=1,2, \cdots),
\end{gathered}
$$

where $\alpha(\theta)$ is as in Theorem 1.

Proof. Similar to the proof of Theorem 8.

In the following three theorems we obtain sharp bounds for the Faber coefficients of functions in the classes $C(E), P(E)$, and $T(E)$. We first need the following two lemmas: 
Lemma 1. If $\alpha(\theta)$ is given by (21), then $\alpha^{\prime}(\theta)$ decreases as $\theta$ increases from 0 to $\pi / 2$.

Lemma 2. If $\alpha(\theta)$ is given by (21), then

$$
\frac{|\sin n \alpha(\theta)|}{\sin \theta} \leq \frac{\pi n}{2 K\left(1-k_{0}\right)}, \quad 0 \leq \theta \leq \frac{\pi}{2}
$$

Proof of Lemma 1. We have from (21)

$$
\frac{2 K}{\pi}\left(\frac{\pi}{2}-\alpha(\theta)-\frac{\pi \tau}{4}\right)=\operatorname{sn}^{-1}\left(\frac{e^{-i \theta}}{\sqrt{k_{0}}}\right)=\int_{0}^{e^{-i \theta} / \sqrt{k_{0}}} \frac{d t}{\sqrt{1-t^{2}} \sqrt{1-k_{0}^{2} t^{2}}} .
$$

Hence we obtain

$$
\alpha^{\prime}(\theta)=\frac{i e^{-i \theta}}{\sqrt{k_{0}}} \frac{\pi}{2 K} \frac{1}{\sqrt{1-\frac{e^{-2 i \theta}}{k_{0}}} \sqrt{1-k_{0} e^{-2 i \theta}}} .
$$

Thus it follows from (31) that

$$
\alpha^{\prime}(\theta)=\frac{\pi}{2 K\left[\left(1-k_{0}\right)^{2}+4 k_{0} \sin ^{2} \theta\right]^{1 / 2}}, \quad 0 \leq \theta \leq \frac{\pi}{2} .
$$

Hence

$$
\alpha^{\prime \prime}(\theta)=-\frac{2 k_{0} \pi \sin 2 \theta}{K\left[\left(1-k_{0}\right)^{2}+4 k_{0} \sin ^{2} \theta\right]^{3 / 2}} \leq 0
$$

since $0 \leq \theta \leq \frac{\pi}{2}$, and Lemma 1 follows.

Proof of Lemma 2. Let

$$
g(\theta)=\alpha^{\prime}(0) \sin \theta-\sin \alpha(\theta), \quad 0 \leq \theta \leq \frac{\pi}{2} .
$$

It follows from Lemma 1 that

$$
g^{\prime}(\theta)=\alpha^{\prime}(0) \cos \theta-\alpha^{\prime}(\theta) \cos \alpha(\theta) \geq \alpha^{\prime}(0)(\cos \theta-\cos \alpha(\theta)) .
$$

Since $\alpha(\theta)$ increases from 0 to $\frac{\pi}{2}$ as $\theta$ increases from 0 to $\frac{\pi}{2}$ and $\alpha^{\prime}(\theta)$ decreases as $\theta$ increases from 0 to $\frac{\pi}{2}$, we have

$$
\alpha(\theta) \geq \theta, \quad 0 \leq \theta \leq \pi / 2 .
$$

Thus it follows from $(61)$ and $(62)$ that $g^{\prime}(\theta) \geq 0$, i.e.

$$
\frac{\sin \alpha(\theta)}{\sin \theta} \leq \alpha^{\prime}(0)=\frac{\pi}{2 K\left(1-k_{0}\right)}
$$

Hence (63) yields

$$
\frac{|\sin n \alpha(\theta)|}{\sin \theta}=\frac{|\sin n \alpha(\theta)|}{\sin \alpha(\theta)} \frac{\sin \alpha(\theta)}{\sin \theta} \leq \frac{n \pi}{2 K\left(1-k_{0}\right)},
$$

which completes the proof of Lemma 2. 
Theorem 10. If $f \in C$ and $c(z)=\frac{z}{1-z}$, then

$$
\left|A_{n}(f)\right| \leq A_{n}(c)=\frac{\pi^{2}}{4 K^{2} \sqrt{k_{0}}\left(1-k_{0}\right)\left(1+2^{-2 n}\right)} \quad(n=0,1,2, \cdots) .
$$

Equality occurs only for the functions $f(z)=c(z)$ and $f(z)=-c(-z)$.

Proof. Using (16) with $\mathcal{F}=C$ and (17), it is enough to show that maximum of $\left|A_{n}\left(c_{\theta}\right)\right|$ over $\theta \in[0,2 \pi)$ occurs at $\theta=0$ and $\theta=\pi$. We will give the proof only for $\theta \in\left[0, \frac{\pi}{2}\right]$. For other values of $\theta$ proofs may be given by using Theorem 2 or 3 or 4 , depending on the quadrant of $\theta$. Using Theorem 1, it suffices to show that

or, equivalently,

$$
\frac{\left(1-2^{-2 n}\right)^{2}+4 \cdot 2^{-2 n} \sin ^{2} n \alpha(\theta)}{\left(1-k_{0}\right)^{2}+4 k_{0} \sin ^{2} \theta} \leq \frac{\left(1-2^{-2 n}\right)^{2}}{\left(1-k_{0}\right)^{2}}
$$

$$
\frac{\sin ^{2} n \alpha(\theta)}{\sin ^{2} \theta} \leq \frac{k_{0} 2^{2 n}\left(1-2^{-2 n}\right)^{2}}{\left(1-k_{0}\right)^{2}} \quad(n=1,2, \cdots) .
$$

It follows from Lemma 2 that

$$
\frac{\sin ^{2} n \alpha(\theta)}{\sin ^{2} \theta} \leq \frac{\pi^{2} n^{2}}{4 K^{2}\left(1-k_{0}\right)^{2}} \quad(n=1,2, \cdots)
$$

Thus the proof is completed if we show that

or

$$
\frac{\pi^{2} n^{2}}{4 K^{2}\left(1-k_{0}\right)^{2}} \leq \frac{k_{0} 2^{2 n}\left(1-2^{-2 n}\right)^{2}}{\left(1-k_{0}\right)^{2}} \quad(n=1,2, \cdots)
$$

$$
\frac{\pi}{2 K \sqrt{k_{0}}} \leq \frac{2^{n}\left(1-2^{-2 n}\right)}{n} \quad(n=1,2, \cdots) .
$$

The sequence $\left\{\frac{2^{n}\left(1-2^{-2 n}\right)}{n}\right\}_{n=1}^{\infty}$ is increasing. Hence

$$
\frac{2^{n}\left(1-2^{-2 n}\right)}{n} \geq \frac{3}{2}>\frac{\pi}{2 K \sqrt{k_{0}}}, \quad(n=1,2, \cdots),
$$

which completes the proof for $n=1,2, \cdots$. We may include $n=0$ since (64) holds trivially in this case.

Theorem 11. If $f \in P$ and $c(z)$ is as in Theorem 10, then

$$
\left|A_{n}(f)\right| \leq 2 A_{n}(c) \quad(n=0,1,2, \cdots) .
$$

Equality occurs only for the functions $f(z)=p(z)$ and $f(z)=p(-z)$, where $p(z)=$ $\frac{1+z}{1-z}$.

Proof. Using (16) with $\mathcal{F}=P$ and (19), it suffices to show that the maximum of $\left|A_{n}\left(p_{\theta}\right)\right|$ occurs at $\theta=0$ and $\theta=\pi$. Hence Theorem 11 follows from Theorems 5 and 10.

Theorem 12. If $f \in T$ and $k(z)$ is the Koebe function, then

$$
\left|A_{n}(f)\right| \leq\left|A_{n}(k)\right|=\frac{\pi^{3} n}{8 K^{3} \sqrt{k_{0}}\left(1-k_{0}\right)^{2}\left(1-2^{-2 n}\right)} \quad(n=1,2, \cdots) .
$$

Equality occurs only for the functions $f(z)=k(z)$ and $f(z)=-k(-z)$. 
Remark. The proof for $n=0$ is given in [7].

Proof. Using (16) with $\mathcal{F}=T$ and (18), it is enough to show that maximum of $\left|A_{n}\left(t_{\theta}\right)\right|$ occurs at $\theta=0$ and $\theta=\pi$. We obtain from Theorem 8 and Lemma 2 that

$$
\begin{aligned}
\left|A_{n}\left(t_{\theta}\right)\right| & \leq \frac{\pi^{3} n}{8 K^{3} \sqrt{k_{0}}\left(1-k_{0}\right)\left(1-2^{-2 n}\right)\left(1+k_{0}^{2}-2 k_{0} \cos 2 \theta\right)^{1 / 2}}, \quad 0<\theta \leq \frac{\pi}{2}, \\
& \leq \frac{\pi^{3} n}{8 K^{3} \sqrt{k_{0}}\left(1-k_{0}\right)^{2}\left(1-2^{-2 n}\right)} .
\end{aligned}
$$

Hence, we have from Theorem 6

$$
\left|A_{n}\left(t_{\theta}\right)\right| \leq A_{n}(k), \quad 0<\theta \leq \frac{\pi}{2} .
$$
way.

The proof for $\frac{\pi}{2}<\theta \leq \pi$ follows from Theorem 9 and Theorem 7 in the same

Note that here we also showed that

$$
\lim _{\theta \rightarrow 0} A_{n}\left(t_{\theta}\right)=A_{n}\left(t_{0}\right)=A_{n}(k)
$$

and

$$
\lim _{\theta \rightarrow \pi} A_{n}\left(t_{\theta}\right)=A_{n}\left(t_{\pi}\right)=A_{n}(-k(-z)) .
$$

As a final note we make the following conjecture:

Conjecture. If $f \in S$, then

$$
\left|A_{n}(f)\right| \leq \frac{\pi^{3} n}{8 K^{3} \sqrt{k_{0}}\left(1-k_{0}\right)^{2}\left(1-2^{-2 n}\right)} \quad(n=1,2, \cdots), \quad f \in S,
$$

and

$$
A_{0}(f) \leq A_{0}(k)=10.5984, \quad f \in S .
$$

This conjecture was proved for the cases $n=0,1,2$ in [7].

\section{ACKNOWLEDGMENT}

The author wishes to express his thanks to Elgin H. Johnston for his guidance and useful suggestions.

\section{REFERENCES}

[1] L. Bieberbach. Über die Koeffizienten derjenigen Potenzreihen, welche eine schlichte Abbildung des Einheitskreises vermitteln. S.-B. Preuss. Akad. Wiss. 1916, 940-955.

[2] L. de Branges. A proof of the Bieberbach conjecture. Acta Math. 154 (1985), 137-152. MR 86h:30026

[3] D. A. Brannan, J. G. Clunie and W. E. Kirwan. On the coefficient problem for functions of bounded boundary rotation. Ann. Acad. Sci. Fenn. Ser. A1 523 (1973), 18 pp. MR 49:3108

[4] L. Brickman, T. H. MacGregor, and D. R. Wilken. Convex hulls of some classical families of univalent functions. Trans. Amer. Math. Soc. 156 (1971), 91-107. MR 43:494

[5] C. Carathéodory. Über den Variabilitätsbereich der Koeffizienten von Pontenzreihen, die gegegbene Werte nicht annehmen. Math. Ann. 64 (1907), 95-115.

[6] P. L. Duren. Univalent functions. Springer-Verlag, New York, 1983. MR 85j:30034

[7] E. Haliloglu. Bounds for Faber coefficients of functions univalent in an ellipse, Ph.D. Thesis, Iowa State University, Ames, IA, 1993.

[8] D. F. Lawden. Elliptic functions and applications. Springer-Verlag, New York, 1989. MR 90h:33001 
[9] K. Loewner. Untersuchungen über die Verzerrung bei konformen Abbildungen des Einheitskreises $|z|<1$, die durch Funktionen mit nichtverschwindender Ableitung geliefert werden. S.-B. Sächs. Akad. Wiss. 69 (1917), 89-106.

[10] Z. Nehari. Conformal mapping. McGraw-Hill, New York, 1952. MR 13:640h

[11] W. W. Rogosinski. Über positive harmonische Entwicklungen und typisch-reelle Potenzreihen. Math. Z., 35 (1932), 93-121.

[12] G. Schober. Univalent functions - selected topics. Lecture Notes in Math., No. 478, SpringerVerlag, New York, 1975. MR 58:22527

Department of Mathematics, Iowa State University, Ames, Iowa 50011

Current address: Department of Mathematics, Istanbul Technical University, Istanbul, Turkey 80626

E-mail address: halilogl@sariyer.cc.itu.edu.tr 